\title{
The International Prospective Glanzmann Thrombasthenia Registry: Pediatric Treatment and Outcomes
} \author{
Thrombasthenia Registry Investigators \\ ${ }^{1}$ Institute for Laboratory Medicine, Blood Coagulation and \\ Transfusion Medicine (LBT), Düsseldorf, Germany \\ 2 Department of Hemostasis, Hemotherapy and Transfusion Medicine, \\ Heinrich Heine University Medical Centre, Düsseldorf, Germany \\ ${ }^{3}$ Departments of Medicine, Pediatrics and Oncology, University of \\ Calgary, Calgary, Alberta, Canada \\ ${ }^{4}$ Southern Alberta Rare Blood and Bleeding Disorders Comprehensive \\ Care Program, Foothills Medical Centre, Calgary, Alberta, Canada \\ ${ }^{5}$ Department of Clinical Medicine and Surgery, Regional Reference \\ Center for Coagulation Disorders, Federico II University, Naples, Italy \\ ${ }^{6}$ Center for Hemophilia and Rare Congenital Bleeding Disorders, \\ University Hospitals Paris-Sud, AP-HP, Bicêtre Hospital, Le Kremlin- \\ Bicêtre, France
}

Rainer B. Zotz ${ }^{1,2}$ Man-Chiu Poon ${ }^{3,4}$ Giovanni Di Minno ${ }^{5}$ Roseline D’Oiron ${ }^{6}$ for the Glanzmann

TH Open 2019;3:e286-e294.
Address for correspondence Priv. Doz. Dr. med. Rainer B. Zotz, Institute for Laboratory Medicine, Blood Coagulation and Transfusion Medicine (LBT), Immermannstr. 65A, 40210 Düsseldorf, Germany (e-mail: zotz@zotzklimas.de).

\section{Abstract \\ Keywords \\ - recombinant activated factor VII \\ - Glanzmann thrombasthenia \\ - autosomal dominant \\ - pediatric \\ - registry \\ - observational study}

Background Standard treatment for Glanzmann thrombasthenia (GT), a severe inherited bleeding disorder, is platelet transfusion. Recombinant activated factor VII ( $r F V I I a)$ is reported to be effective in GT with platelet antibodies and/or refractoriness to platelet transfusions.

Methods We evaluated rFVIla effectiveness and safety for the treatment and prevention of surgical and nonsurgical bleeding in children $<18$ years old, with or without platelet antibodies and/or refractoriness, as reported in the GT Registry (GTR). Data were used from the GTR, an international, multicenter, observational, postmarketing study of rFVIla that prospectively collected data on the treatment and outcomes of bleeds in patients with GT. Only patients with a diagnosis of congenital GT were included in the registry.

Results Between 2007 and 2011, 27 children were treated for 44 surgical procedures (minor: 36; major: 8); nonsurgical bleeds occurred in 104 patients (599 episodes: severe, 145; moderate, 454; spontaneous, 423; posttraumatic, 176). The effectiveness of treatment for minor procedures, major procedures, nonsurgical bleeds was $6 / 6,1 / 1$, and $75 / 84$ for rFVIla, 6/6, 2/2, and 64/76 for rFVIla + antifibrinolytics (AF), 11/12, 1/1, and $162 / 214$ for platelets $\pm A F$, and $5 / 6,0 / 3$, and $33 / 45$ for rFVIla + platelets $\pm A F$. In all, 25 adverse events were reported in children; no thromboembolic events were reported.

Conclusion For all patients, regardless of platelet antibody or refractoriness status, rFVIla, administered with or without platelets ( \pm AF), provided effective hemostasis with a low frequency of adverse events in surgical, as well as nonsurgical, bleeding in patients with GT.

clinicaltrials.gov identifier: NCT01476423. received

February 4, 2019

accepted after revision

July 25, 2019
DOI https://doi.org/

10.1055/s-0039-1696657.

ISSN 2512-9465. (c) 2019 Georg Thieme Verlag KG Stuttgart · New York
License terms

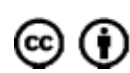




\section{Introduction}

Glanzmann thrombasthenia (GT) is an autosomal platelet function disorder caused by a quantitative or qualitative defect of the platelet membrane glycoprotein IIb-IIla (integrin $\alpha$ IIb $\beta 3$ ) complex. ${ }^{1,2}$ With a prevalence of $1: 1$ million, GT is rare, although the prevalence is higher in areas where marriage between relatives is common. It manifests clinically as an increased tendency to spontaneous bleeds (e.g., epistaxis, hematoma, menorrhagia, and bleeding complications) and also during and after surgery. ${ }^{2,3}$

The standard treatment for GT is platelet transfusion (PT), which carries the risk of development of antibodies $(\mathrm{AB})$ to $\alpha I I b \beta 3$ and/or human leukocyte antigen (HLA). Possible alternatives to PT include the administration of antifibrinolytics (AF), local hemostatic agents, and bone marrow transplantation. In addition, recombinant activated factor VII (rFVIIa) can be used for the treatment and prevention of bleeding episodes, and for surgery, in patients with GT and AB, as well as past or present platelet refractoriness. Nevertheless, this off-label use of rFVIIa in patients with GT who have bleeds is increasing, mainly because of its convenience and to avoid alloimmunization to platelets. Data from an international survey of patients with GT who have bleeding episodes or hemorrhagic surgery complications supported a preliminary suggestion of an optimal rFVIIa dosing regimen for the treatment of moderate or severe bleeding episodes ( $\geq 80 \mu \mathrm{g} / \mathrm{kg}$ given at intervals of $\leq 2.5$ hours for $\geq 3$ doses). ${ }^{4}$ Based on these survey results, rFVIIa was approved by the European Medicines Agency (EMA) in 2004 for use in patients with GT who have $A B$ and past or present platelet refractoriness.

As the prevalence of GT is so low, data are not readily available for different subgroups of patients. Particularly rare are data on the treatment of GT in children, for both surgical and nonsurgical bleeding; though case studies are available, no large pediatric studies have been conducted. The GT Registry (GTR) was an international, multicenter, observational registry that focused on both adults and children. As such, it is one of the first efforts to provide pediatricians with comprehensive information on the effectiveness and safety of rFVIIa in children. This article summarizes new GTR data and aims to evaluate rFVIIa effectiveness and safety as a treatment for young patients with GT. Treatment results for all GTR patients have been published previously.,

\section{Methods}

The GTR was an international, multicenter, observational study on the effectiveness and safety of rFVIIa in patients with GT (ClinicalTrials.gov identifier: NCT01476423), prospectively collecting data on the treatment and outcomes of bleeds. Data were entered into the GTR from 2007 until its closure in 2011, using a customized, Web-based collecting tool. Treatment was based on local clinical practice rather than a set protocol, and performed in accordance with general data protection laws and local country requirements for conducting observational studies. ${ }^{5,6}$ Centralized data management was overseen by Novo Nordisk and an external expert panel comprising four hemostasis physicians from Europe and North America.

\section{Patients}

The analyses focused on patients $<18$ years old, and only a diagnosis of congenital GT was included in the GTR. Patients with acquired thrombasthenic states caused by autoimmune disorders or medications were excluded. Refractoriness and the presence of $A B$ were coded initially and assessed periodically as deemed necessary by the investigator. Because tests for $A B$ may not have been available at all centers, $A B$ may also have been present in patients classified as having refractoriness only. For GT definitions, as well as key terms regarding bleeding, surgery, and definitions of effectiveness (effective, partially effective, ineffective), see $\boldsymbol{\sim}$ Supplementary Table $\mathbf{S 1}$.

\section{Ethics Committee Approvals}

The GTR was conducted in accordance with the Declaration of Helsinki and Guidelines for Good Pharmacoepidemiology Practices. Each participating center complied with local regulations. Ethical and/or regulatory approval was obtained before data entry into the registry, as required. Signed informed consent to participate was obtained from all parents/legal guardians of the patients.

\section{Statistical Methods}

The effectiveness analysis was based on all children in the GTR and treatment-allocated bleeds for which the efficacy endpoint was known. All children and bleeding episodes were included in the safety analysis. The effectiveness data were summarized using numerical variables (mean, standard deviation, median, maximum, and minimum), while categorical variables were summarized as numbers and percentages. No formal statistical comparisons were performed. No subdivision into age groups was performed because patient numbers would be too low to yield meaningful results.

\section{Results}

\section{GTR Enrollment and Composition of Datasets}

Details of recruitment into the GTR and the safety and effectiveness datasets for all surgical and nonsurgical bleeds have been reported previously for adults and children. ${ }^{5,6}$ For children with GT, data were collected from 643 admissions (bleeding, 599; surgery, 44); 131 children with GT were enrolled from 45 sites in 15 countries from Africa, Asia, Europe, and North America. The safety analysis dataset included all 643 admissions, while effectiveness analyses were performed using data available from 590 admissions for nonsurgical bleeding and 44 surgical admissions.

\section{Clinical and Demographic Characteristics of the GTR Population Undergoing Surgery or with Nonsurgical Bleeds}

The clinical and demographic characteristics of children who participated in the GTR and underwent surgery are provided in - Table 1. Of 44 invasive procedures reported in 27 patients, 36 (in 23 patients) were minor and 8 (in 8 patients) were major. 
Table 1 Registry: clinical and demographic characteristics of the population $<18$ years old at admission

\begin{tabular}{|c|c|c|c|c|}
\hline Variable & $\begin{array}{l}\text { Nonsurgical } \\
\text { patients } \\
(n=104)\end{array}$ & $\begin{array}{l}\text { Nonsurgical } \\
\text { bleeding episodes } \\
(n=599)\end{array}$ & $\begin{array}{l}\text { Surgical } \\
\text { patients } \\
(n=27)\end{array}$ & $\begin{array}{l}\text { Surgical } \\
\text { episodes } \\
(n=44)\end{array}$ \\
\hline Males, $n(\%)$ & $48(46.2)$ & $259(43.2)$ & $19(70.4)$ & $34(77.3)$ \\
\hline Females, $n(\%)$ & $56(53.9)$ & $340(56.8)$ & $8(29.6)$ & $10(22.7)$ \\
\hline Age $(y)$, mean $\pm S D$ & $6.3 \pm 4.8$ & $6.6 \pm 4.3$ & $7.5 \pm 4.5$ & $7.9 \pm 4.4$ \\
\hline Males, mean $\pm S D$ & $5.2 \pm 4.4$ & $5.1 \pm 4.3$ & $6.3 \pm 4.0$ & $7.2 \pm 4.3$ \\
\hline Females, mean $\pm S D$ & $7.2 \pm 5.0$ & $7.7 \pm 4.0$ & $10.3 \pm 4.8$ & $10.0 \pm 4.4$ \\
\hline \multicolumn{5}{|l|}{ Age category, $n(\%)$} \\
\hline$<12$ years & $87(83.7)$ & $506(84.5)$ & $21(77.8)$ & $34(77.3)$ \\
\hline $12-17$ years & $17(16.4)$ & $93(15.5)$ & $6(22.2)$ & $10(22.7)$ \\
\hline Body weight $(\mathrm{kg})$, mean $\pm \mathrm{SD}$ & $21.2 \pm 14.3$ & $22.3 \pm 13.3$ & $30.3 \pm 20.8$ & $31.6 \pm 20.8$ \\
\hline Males, mean \pm SD & $19.3 \pm 14.5$ & $20.0 \pm 14.1$ & $26.3 \pm 17.1$ & $30.0 \pm 19.8$ \\
\hline Females, mean $\pm S D$ & $22.8 \pm 14.0$ & $24.2 \pm 12.4$ & $39.9 \pm 26.6$ & $37.0 \pm 24.2$ \\
\hline \multicolumn{5}{|l|}{ Type of disease, $n(\%)$} \\
\hline Type 1 & $26(25.0)$ & $234(39.1)$ & $9(33.3)$ & $13(29.6)$ \\
\hline Type 2 & $12(11.5)$ & $34(5.7)$ & $1(3.7)$ & $1(2.3)$ \\
\hline Variant & $3(2.9)$ & $8(1.3)$ & - & - \\
\hline Unknown type & $63(60.6)$ & $323(53.9)$ & $17(63.0)$ & $30(68.2)$ \\
\hline \multicolumn{5}{|l|}{ History of antiplatelet $A B$ and/or refractoriness to platelets } \\
\hline$A B$ ( $A B$ confirmed, refractoriness status unknown) & $11(10.6)$ & $85(14.2)$ & $5(18.5)$ & $12(27.3)$ \\
\hline$A B+$ refractoriness (both confirmed) & $5(4.8)$ & 77 (12.9) & $4(14.8)$ & $5(11.4)$ \\
\hline All $A B^{a}$ & 16 & 162 & 9 & 17 \\
\hline Anti- $\alpha$ Ilb $\beta 3$ & $13(81.3)$ & $64(39.5)$ & $7(77.8)$ & $13(76.5)$ \\
\hline Antihuman leukocyte antigen & $2(12.5)$ & $9(5.6)$ & $1(11.1)$ & $3(17.7)$ \\
\hline Other & $5(31.3)$ & $56(34.6)$ & $2(22.2)$ & $3(17.7)$ \\
\hline $\begin{array}{l}\text { Refractoriness } \\
\text { (refractoriness confirmed, AB not confirmed) }\end{array}$ & $3(2.9)$ & $37(6.2)$ & $1(3.7)$ & $1(2.3)$ \\
\hline $\begin{array}{l}\text { No confirmed history of antiplatelet } \\
A B \text { and/or refractoriness to PT }\end{array}$ & $85(81.7)$ & $400(66.8)$ & $17(63.0)$ & $26(59.1)$ \\
\hline
\end{tabular}

Abbreviations: $A B$, antibodies; PT, platelet transfusions; $S D$, standard deviation.

${ }^{a}$ Number of types of $A B$ may not add up to number of all $A B$, since type of $A B$ was not always registered, or more than one type of $A B$ was registered.

Of the 599 admissions for nonsurgical bleeds in children, 145 (24.2\%) were classified as severe and 454 (75.8\%) as moderate, while 423 bleeds (70.6\%) were spontaneous and 176 (29.4\%) were classified as being posttraumatic (see - Supplementary Table S2 for a detailed description of the bleeding episodes reported). Clinical and demographic characteristics of the patients who experienced nonsurgical bleeds are presented in -Table 1.

\section{Surgical Bleeding-Minor Procedures}

\section{Treatment and Outcome}

Of the 36 minor surgical procedures performed, dental procedures were most common $(25 / 36 ; 69.4 \%)$, followed by nasal procedures $(4 / 36 ; 11.1 \%)$. Most minor procedures (-Table 2A) were treated with rFVIIa, either alone (6/36; $16.7 \%)$, with AF $(6 / 36 ; 16.7 \%)$, or PTs $(12 / 36 ; 33.3 \%)$. Data on the number of minor procedures rated as "effective" (see definition in -Supplementary Table S1) for the different treatments (overall and stratified according to the status of platelet $\mathrm{AB}$ and platelet refractoriness) are provided in -Table $\mathbf{2 A}$.

\section{Treatment with rFVIla}

Data on rFVIIa use were available for 18 minor procedures treated with rFVIIa, rFVIIa + AF, or rFVIIa + PT \pm AF; $100 \%$ of treatments were rated effective for rFVIIa (6/6) and rFVIIa $+\mathrm{AF}(6 / 6)$, and $83.3 \%$ (5/6) of treatments were rated effective for rFVIIa $+\mathrm{PT} \pm \mathrm{AF}$ (-Table $\mathbf{2 A}$ ). For all minor procedures, median rFVIIa dose was $175 \mu \mathrm{g} / \mathrm{kg}$ (range: 3.6-300; interquartile range [IQR]: $110 \mu \mathrm{g} / \mathrm{kg}$ ), median number of doses was two (range: 1-20; IQR: 2) and median interval between doses was 2 hours (range: 2-6; IQR: 1 hour) (-Table 3). 
Glanzmann Thrombasthenia Pediatric Registry Zotz et al. e289

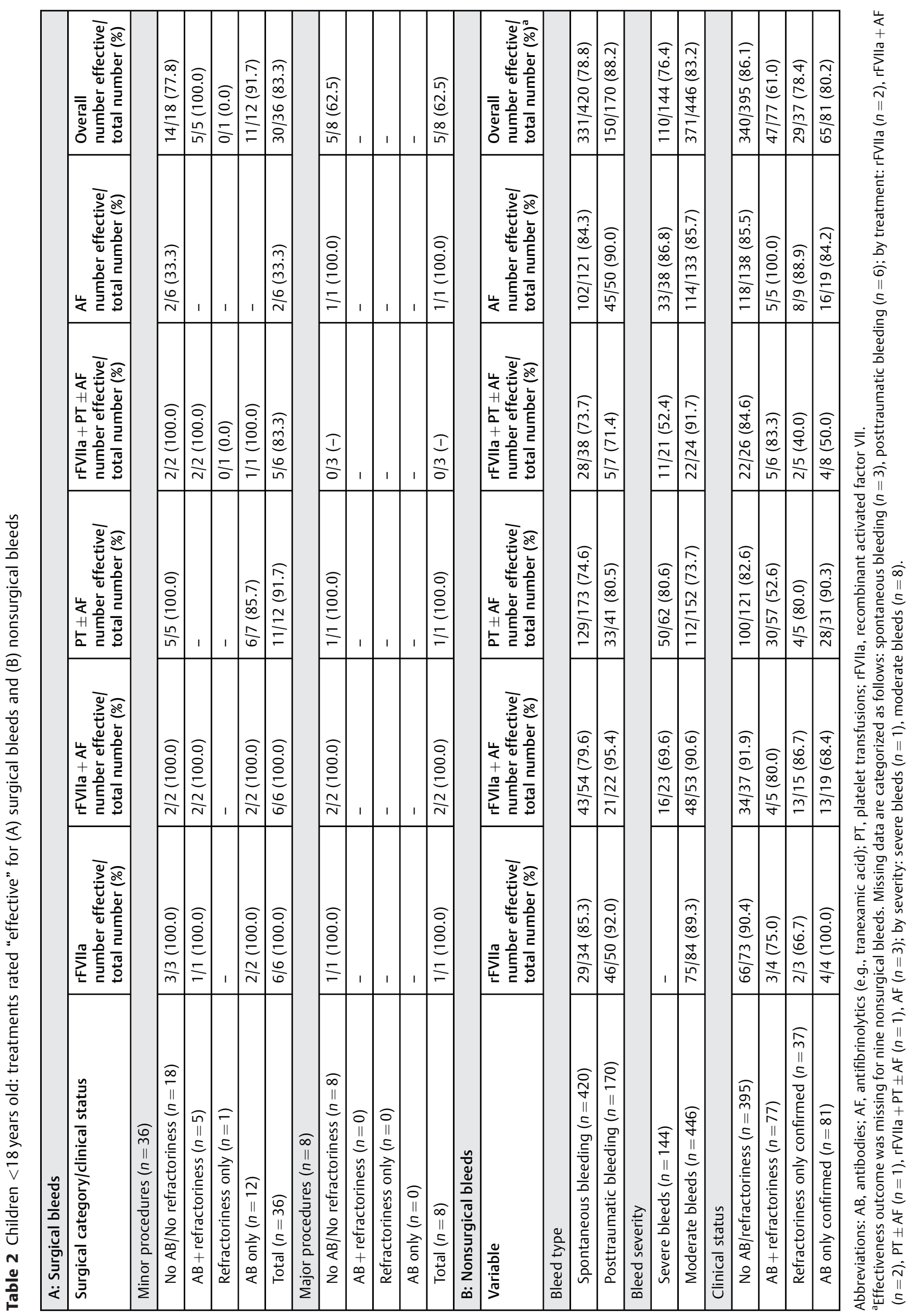




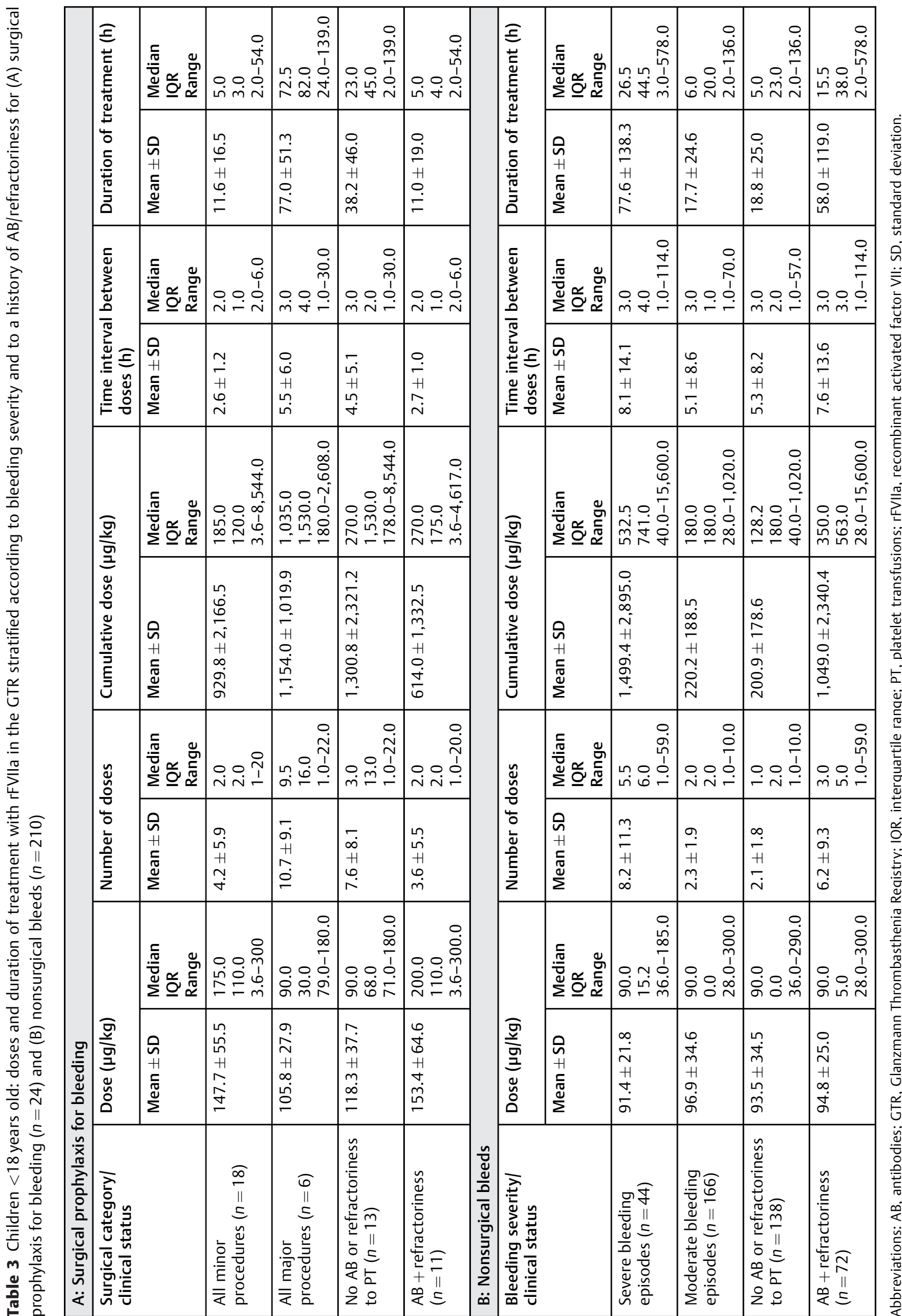




\section{Treatment with Platelets}

Treatment with $\mathrm{PT} \pm \mathrm{AF}$ was used in 12 minor procedures, with $91.7 \%$ of treatments overall being rated effective (-Table 2A). This treatment was rated effective in all five procedures in the no $A B /$ no refractoriness group and in six of seven procedures in the AB-only group. For the six minor procedures treated with $\mathrm{FFVIIa}+\mathrm{PT} \pm \mathrm{AF}, 83.3 \%$ were rated effective overall ( $\mathbf{-}$ Table $\mathbf{2 A}$ ).

\section{Ineffective Treatments}

One of the 36 minor procedures was rated "ineffective" (no $\mathrm{AB} /$ no refractoriness; $\mathrm{AF}$ only) using $\mathrm{PT} \pm \mathrm{AF}$.

\section{Surgical Bleeding-Major Procedures}

\section{Treatment and Outcome}

Of the eight major surgical procedures performed, gastrointestinal and circumcision procedures were most common ( $n=3 ; 37.5 \%$ each). Major procedures were treated most frequently with rFVIIa $+\mathrm{PT} \pm \mathrm{AF}(n=3 ; 37.5 \%)$. All major procedures occurred in the no $\mathrm{AB} /$ no refractoriness group. Effectiveness ratings for the different treatments for major procedures (overall and stratified according to the status of platelet $\mathrm{AB}$ and platelet refractoriness) are provided in - Table $\mathbf{2 A}$.

\section{Treatment with rFVIIa}

Data on rFVIIa use were available for analysis in six major procedures treated with rFVIIa, rFVIIa $+\mathrm{AF}$, or rFVIIa $+\mathrm{PT} \pm \mathrm{AF}$, with $100 \%$ of treatments rated effective for rFVIIa (1/1) and rFVIIa $+A F(2 / 2) ; 0 \%(0 / 3)$ of treatments were rated effective for rFVIIa $+\mathrm{PT} \pm \mathrm{AF}$ (- Table 2A). For all major procedures, the median rFVIIa dose was $90 \mu \mathrm{g} / \mathrm{kg}$ (range: 79-180); median dose interval was 3 hours (range: $1-30$ hours). The median number of doses was 9.5 (range: 1-22) and the median cumulative rFVIIa dose was $1,035 \mu \mathrm{g} / \mathrm{kg}$ (range: 180-2,608) (-Table 3).

\section{Treatment with Platelets}

The effectiveness outcome for major procedures treated with platelets (PT $\pm \mathrm{AF}$ and $\mathrm{rFVIIa}+\mathrm{PT} \pm \mathrm{AF}$ ) was available for four cases ( - Table $2 A$ ). Of these, the one treated with $\mathrm{PT} \pm \mathrm{AF}$ was effective.

\section{Partially Effective/Ineffective Treatments}

Of the eight major procedures, three were rated as either partially effective $(n=2)$ or ineffective $(n=1)$ in patients treated with rFVIIa $+\mathrm{PT} \pm \mathrm{AF}$ with no $\mathrm{AB} /$ refractoriness. No postsurgical bleeding was reported following rFVIIa and/or platelet treatment for major procedures (as defined in - Supplementary Table S1).

\section{Safety Data}

For surgery, one nonserious adverse event (AE) was reported in the GTR, which was an incidence of pyrexia in a male child without platelet $A B$ or refractoriness, who was treated with rFVIIa + PT + AF (major surgery). It was considered unlikely that this AE was related to rFVIIa, and the patient recovered completely. - Supplementary Table $\mathbf{5 3}$ summarizes the AEs reported in treated patients.

\section{Nonsurgical Bleeding}

\section{Treatment Dosing and Scheduling}

Regardless of bleeding severity, the $\mathrm{PT} \pm \mathrm{AF}$ combination was the most commonly used treatment (-Supplementary Fig. S1). Of the 590 bleeding episodes included in the effectiveness assessment, 214 (36.3\%) were treated with $\mathrm{PT} \pm \mathrm{AF}, 171$ (29.0\%) with AF only, 84 (14.2\%) with rFVIIa alone, 76 (12.9\%) with rFVIIa + AF, and 45 (7.6\%) with rFVIIa $+\mathrm{PT} \pm \mathrm{AF}$. Concomitant AF treatment was documented in 76/ 160 (47.5\%) bleeds treated with rFVIIa, in 60/214 (28.0\%) treated with PT, and in $36 / 45$ (80.0\%) treated with rFVIIa + PT.

Investigators reported that, for the $195 / 590$ bleeds that occurred in patients with a history of antiplatelet $A B$ and platelet refractoriness, $\mathrm{AB}$ only, or refractoriness only, $\mathrm{PT} \pm$ AF was used in 93/195 (47.7\%; 29 severe and 64 moderate), rFVIIa + AF in 39/195 (20.0\%, 20 severe and 19 moderate), AF alone in 33/195 (16.9\%, 2 severe and 31 moderate), rFVIIa + $\mathrm{PT} \pm \mathrm{AF}$ in 19/195 (9.7\%, 15 severe and 4 moderate), and rFVIIa alone in $11 / 195$ (5.6\%, all moderate) bleeds. The use of rFVIIa, alone $(3 / 37 ; 8.1 \%)$ or together with $\mathrm{AF}(15 / 37 ; 40.5 \%)$, to treat bleeds was most frequent in patients with a history of refractoriness alone, while AF was mainly used to treat bleeds that occurred in patients with no history of $A B$ and/ or platelet refractoriness (138/395; 34.9\%) (-Table 2B).

The number of rFVIIa doses, cumulative dose, and overall duration of treatment were greater in patients with severe bleeds than in those with moderate bleeds. They were also greater in patients with a history of $A B$ and/or refractoriness to platelets versus patients without such medical history (-Table 3).

\section{Evaluation of Treatment Effectiveness}

Overall, treatment was judged as effective (see - Supplementary Table S1 for definition) in 89.3\% (75/84) of bleeds treated with rFVIIa alone, $86.0 \%(147 / 171)$ with AFalone, 84.2\% (64/76) with rFVIIa + AF, 75.7\% (162/214) with PT \pm AF, and $73.3 \%$ (33/45) with rFVIIa $+\mathrm{PT} \pm \mathrm{AF}$ (-Table 2B). For 73 moderate bleeds treated with rFVIIa (either alone, with AF, or with PT) for which information on treatment duration was available, the median total duration of rFVIIa treatment was 6 hours. For 36 severe bleeds treated with rFVIIa (either alone, with $\mathrm{AF}$, or with PT) for which information on treatment duration was available, the median total duration of rFVIIa treatment was 26.5 hours. In addition, the median total duration of treatment was 4 hours for 20 bleeds with $A B, 11$ hours for 13 bleeds with $A B+$ refractoriness, 5 hours for 57 bleeds without $A B$ or refractoriness, and 34 hours for 19 bleeds with refractoriness.

\section{Ineffective Treatment}

Ineffective treatment was documented in 13 cases $(2.2 \%$ of the total number of patients), comprising three severe bleeds and 10 moderate bleeds. Of these, seven had a history of $\mathrm{AB} /$ refractoriness. Regarding the initial treatment employed, 8 of the 13 cases were receiving $A F, 3$ were receiving $\mathrm{PT} \pm \mathrm{AF}$, and 2 were receiving $\mathrm{rFVIIa}+\mathrm{AF}$. 


\section{Rebleeding}

For 557 bleeds where data on rebleeding were available, 36 rebleeds in 17 patients were registered, comprising 15/136 (11.0\%) severe bleeds, 21/421 (5.0\%) moderate bleeds, $15 / 380$ (3.9\%) bleeds in patients without $A B$ or refractoriness, and $21 / 177$ (11.9\%) bleeds in patients with a history of $A B$ and/or refractoriness to platelets. Rebleeding occurred in 20/204 (9.8\%) bleeds treated with $\mathrm{PT} \pm \mathrm{AF}, 9 / 42$ (21.4\%) treated with rFVIIa $+\mathrm{PT} \pm \mathrm{AF}, 1 / 78$ (1.3\%) treated with rFVIIa alone, and 3/69 (4.3\%) treated with rFVIIa + AF.

\section{Safety Data}

For the nonsurgical bleeding results presented in this article, 24 AEs were reported (including six serious AEs; - Supplementary Table S3). For bleeds where patients received rFVIla, eight AEs occurred, five of which were serious (subarachnoid bleeding, septicemia, respiratory insufficiency, cardiac decompensation, and rebleeding/hematoma due to a fall) and three nonserious (bacterial infection, fever, and headache). All were judged by the investigators as unlikely to be related to rFVIIa treatment. For the subarachnoid bleed, it was not possible to confirm the time relationship between this serious $\mathrm{AE}$ and rFVIIa treatment, i.e., whether rFVIIa was used to treat the subarachnoid bleed or not, and whether this is related to a lack of efficacy for rFVIIa or not. No thromboembolic events or unexpected laboratory values were reported for any of the treatments for nonsurgical bleeds.

\section{Discussion}

The GTR data reported here have been taken from the largest observational study on patients with GT and include information on the management of invasive procedures; the shortcomings of the previous survey ${ }^{4}$ have been addressed through consideration of the use of hemostatic agents other than rFVIIa. For the most part, this subgroup analysis confirmed previous insights. rFVIIa and other currently available treatments for bleeding in patients with GTwere found to have good safety and effectiveness profiles in most children, in both surgical and nonsurgical bleeds. When compared with previous GTR analyses, overall there were no relevant differences between children and adults regarding effectiveness and safety of the studied treatment options (with the exception of PT for treatment of nonsurgical bleeds in children with $A B$ and refractoriness, which was less effective than in adults). ${ }^{5,6}$ The surgical patient subgroups included in the discussion below (e.g., patients with or without $\mathrm{AB} /$ refractoriness) were based on low patient numbers (-Table 2 ).

\section{Treatment with rFVIla}

In general, rFVIIa, alone or with AF, was used more frequently than platelets $(\mathrm{PT} \pm \mathrm{AF})$ in surgical patients with $\mathrm{AB}$ and refractoriness, in surgical patients with major procedures, in nonsurgical patients with refractoriness only, and in nonsurgical patients with posttraumatic bleeding. The numbers receiving $\mathrm{rFVIIa} \pm \mathrm{AF}$ were similar in both surgical patients without $\mathrm{AB} /$ refractoriness and those with minor procedures overall. In all other subgroups, rFVIIa, alone or with AF, was used less often than platelets. The GTR results further indicate that rFVIIa has a good safety profile in patients with GT.

Assessment of rFVIla dose and dosing schedule in this registry suggests that, in patients without $A B$ or refractoriness, rFVIIa 90 to $120 \mu \mathrm{g} / \mathrm{kg}$ given at approximately 2 to 4 -hour intervals (median: $2-3$ hours) for $\geq 3$ doses (median: $1-5.5$, until effective hemostasis) could be used, with the first dose given immediately preoperatively in surgical patients. As reported by Poon et al, ${ }^{6}$ this is similar to the regimen previously suggested for bleeding episodes (rFVIIa $\geq 80 \mu \mathrm{g} / \mathrm{kg}$ at intervals of $\leq 2.5$ hours for $\geq 3$ doses), ${ }^{4}$ and is also similar to standard rFVIIa dosing in patients who have congenital hemophilia A or B with inhibitors. ${ }^{7}$

A median rFVIIa dose of $90 \mu \mathrm{g} / \mathrm{kg}$ was used most frequently in nonsurgical patients with $\mathrm{AB}$ and/or refractoriness, and in surgical patients with neither of these $(200 \mu \mathrm{g} / \mathrm{kg}$ was used in surgical patients with $A B$ and/or refractoriness). The median dose interval was 3 hours, except in surgical patients with $A B$ and/or refractoriness (for whom it was 2 hours). For severe bleeds, the number of doses reported was understandably higher than for moderate bleeds. In addition, severe bleeds were also associated with longer treatment duration and higher cumulative doses. Based on these results, rFVIIa at $\geq 90 \mu \mathrm{g} / \mathrm{kg}$ at intervals of $\leq 3$ hours should be used, at least at the beginning of treatment. The subsequent number of doses required would need to be determined by the clinical situation and dosing should be continued until hemostasis is assured.

\section{Treatment with Platelets}

PT was generally effective in covering surgical procedures and nonsurgical bleeds in patients, both with and without historic report of platelet $A B$ and/or refractoriness. An exception is the much lower response rate for nonsurgical bleeds in children with $A B$ and refractoriness (30/57,52.6\%) when compared with adults with $A B$ and refractoriness (25/28, 89.3\%). ${ }^{5}$ Notably, some procedures in the AB-only group, as well as some nonsurgical bleeding episodes in patients with $A B$ and/or refractoriness, had a successful outcome with $\mathrm{PT} \pm \mathrm{AF}$ (-Table 2). A possible explanation for this is that, in some patients with a history of $A B$, they were no longer present at the time of surgery and patients who had been refractory before were no longer. As discussed by Di Minno et $a^{5},{ }^{5}$ although comprehensive data on the natural history of inhibitors in GT are lacking, it is possible that the effectiveness of platelets in such a setting may be associated with the long time intervals between exposure to platelets in GT and possibly also to the transient nature of the inhibitors. Furthermore, Poon et $\mathrm{al}^{6}$ suggested that the reason for this effectiveness may be that patients with HLA AB were receiving only HLA-matched platelets. Nevertheless, even if HLA-unmatched platelets are provided, prior data indicate that $>50 \%$ of patients may not display refractoriness to $\mathrm{PT}^{8-10}$ As previously noted for the GTR in surgical intervention, ${ }^{6}$ these observations suggest that treatment with platelets may be attempted when other agents are ineffective or not available-even in patients with a history of $\mathrm{AB}$ and/or refractoriness. ${ }^{4}$ Due to the low numbers of surgical patients available, we cannot provide information on the 
effectiveness of treatment in refractory patients. Successful PT following removal of platelet $A B$ by plasmapheresis ${ }^{11,12}$ or immunoadsorption ${ }^{13}$ has also been reported.

\section{Combined Treatment Using Platelets and rFVIla}

A key question is whether combined use of platelets and rFVIIa (rFVIIa $+\mathrm{PT} \pm \mathrm{AF}$ ) has an advantage over rFVIIa (alone or with $\mathrm{AF}$ ) or $\mathrm{PT} \pm \mathrm{AF}^{4}$ This pediatric analysis correlated with prior data reported from the GTR indicating that $\mathrm{rFVIIa}+\mathrm{PT} \pm \mathrm{AF}$ was less effective than rFVIIa and rFVIIa $+\mathrm{AF}^{6}$ As previously suggested, based upon the observational nature of the registry, ${ }^{6}$ rFVIIa $+\mathrm{PT} \pm \mathrm{AF}$ may have been used in patients with particularly difficult or challenging clinical situations and often when platelets may have been added to rFVIIa $\pm \mathrm{AF}$ or rFVIIa added to $\mathrm{PT} \pm \mathrm{AF}$. Further hemostatic agent(s) might have been added to the regimen when effectiveness was in doubt while using other hemostatic agents.

\section{Treatment with AF}

The relative proportion of AF use for bleeds (both moderate and severe), as well as for surgical prophylaxis (both minor and major), was similar in both children and adults. ${ }^{5,6}$ Nevertheless, in general, children with surgical procedures were administered AF more often than adults (7/44 [15.9\%] vs. 5/159 [3.1\%], respectively); rFVIIa was administered less often in children than adults (15/44 [34.1\%] vs. 118/159 [74.2\%], respectively). ${ }^{6}$ For the treatment of bleeding episodes in particular, children with refractoriness or with $\mathrm{AB}$ were treated more often with $\mathrm{AF}$ than adults with the same status (refractoriness: 9/37 [24.3\%] children vs. 0/16 [0\%] adults; AB: 19/81 [23.5\%] children vs. $11 /$ 52 [21.2\%] adults). ${ }^{5}$ AF were also used in children with spontaneous severe bleeding more often than in adults (36/131 [27.5\%] vs.9/67 [13.4\%], respectively), although both subgroups were treated most often with PT (52/131 [39.7\%] vs. 34/67 [50.7\%], respectively). ${ }^{5}$

The successful use of AF without platelets or rFVIIa was reported in 3/7 (42.9\%) surgical procedures, and in 147/171 (86.0\%) nonsurgical bleeding episodes. As noted previously for the GTR, ${ }^{6}$ this may be explained by AF treatment commencing with the intention of using other systemic hemostatic agent(s), should hemostasis not be achieved. In addition, a less controlled data collection process versus clinical trials complicates interpretation or direct comparison of effectiveness between treatments. We do advise, however, that AF should not be recommended as the sole therapy during surgery in pediatric patients, particularly for major procedures, unless rFVIIa or platelet concentrates are available as back up. As noted by $\mathrm{Di}$ Minno et $a{ }^{5}{ }^{5}$ nonsurgical bleeds may, on the other hand, be treated with AF first (possibly in the home setting), with the option of introducing other treatment (rFVIIa and/or platelets) during the bleeding episode if required.

\section{Safety}

A potential concern with the use of rFVIIa in patients with GT is whether rFVIIa is thrombogenic. The pediatric data reported here suggest that all systemic hemostatic agents used had a good safety profile in surgical procedures for these patients. Among the 599 nonsurgical bleeding epi- sodes in 104 patients and 44 procedures performed in 27 patients, no thromboembolic events were reported. Thus, rFVIIa appears to be a valid first-line treatment option for nonsurgical bleeds in pediatric patients with GT who are awaiting HLA-compatible platelet concentrates or for concentrates prepared from single-donor apheresis. Indeed, rFVIIa has been licensed for use in GT when platelets are not readily available. ${ }^{14}$

\section{Limitations}

As previously reported, ${ }^{6}$ the major limitations of these GTR data are that they were not obtained using a defined treatment protocol in a randomized manner and treatment effectiveness and safety were not assessed at multiple, consistent, predefined time points. Furthermore, the frequent use of multiple agents in GT and delays in obtaining platelets make it particularly difficult to attribute effectiveness to any one or more products. Previously, Di Minno et $\mathrm{al}^{5}$ noted the arbitrary classification of severe and moderate bleeds in nonsurgical bleeding as a further limitation. The use of these simple definitions, which are similar to the definitions of "major and minor bleeds" published in April 2005 by the Subcommittee on Control of Anticoagulation of the Scientific and Standardization Committee of the International Society on Thrombosis and Haemostasis, ${ }^{15}$ was at the request of the EMA. Furthermore, as the coding of history of $A B$ or refractoriness was performed at first admission and when the investigator considered appropriate, ${ }^{6}$ the lack of documentation of specific antibody testing or refractoriness at the time of a particular episode limits the analysis (particularly for use of platelet-based regimens).

The rarity of GT also hinders the performance of formal clinical trials; pediatric data reported here represent the largest dataset available in the literature, including those in other databases. ${ }^{16,17}$ These registry data represent real-life clinical practice and the standards of care at participating sites. Narrative information, as provided in the GTR, often gives additional useful insights that may differ from the coding of effectiveness at an earlier time point after surgery.

In summary, this post hoc analysis suggests that, when managing children with GT and surgical or nonsurgical bleeding episodes, rFVIIa, PT, and AF are valid first-line treatment options as they appear effective and safe. Given the selection bias in choice of treatment and adaption of treatment to clinical response, treatment strategies cannot be compared in observational studies. For first-line treatment of nonsurgical bleeds with AF, we suggest introducing other treatment options if AF proves insufficiently effective. For major surgical procedures, AF should only be used as a first-line therapy when rFVIIa or platelet concentrates are available as a backup treatment option. In cases of severe bleeding, a combined treatment with AF, rFVIIa, and platelet concentrates may be necessary. The available observations suggest that treatment with platelets may be attempted when other agents are ineffective or not available-even in patients with a history of $A B$ and/or refractoriness. As there were no relevant differences between children and adults regarding effectiveness and safety of the studied treatment 
options (with the exception of PT in the treatment of nonsurgical bleeds in children with both $A B$ and refractoriness), the treatment of children should not differ notably from that of adults. ${ }^{5,6}$

\section{Authors' Contributions}

R. B. Zotz drafted, reviewed, and approved the article. M.C. Poon, G. Di Minno, and R. d'Oiron reviewed and approved the article content.

\section{Data Sharing Statement}

The trial sponsor's policy on data sharing may be found at https://www.novonordisk-trials.com/how-access-clinical-trial-datasets.

\section{Conflict of Interest}

R. B. Zotz has been a speaker for AstraZeneca, Bayer, Biotest, Boehringer Ingelheim, CSL Behring, GlaxoSmithKline, LEO Pharma, MEDA, Novartis, Novo Nordisk, Octapharma, Pfizer, Sanofi Aventis, and Wyeth, and received fees from Biotest, CSL Behring, GlaxoSmithKline, Novartis, Sanofi Aventis, and Wyeth. He is a member of the advisory boards at Bayer, Novo Nordisk, and Pfizer. He received research funding from CSL Behring. M.-C. Poon was chair of Novo Nordisk's expert panel on the GTR, has been an ad hoc speaker for Bayer, Novo Nordisk, and Pfizer, and attended advisory board meetings of Bioverativ/Biogen Idec, CSL Behring, Novo Nordisk, Octapharma, Pfizer, Roche and Shire/Baxter, and received grant funding from Bayer and CSL Behring. G. Di Minno has been a speaker or a member of a speakers' bureau for Bayer, Biotest, Boehringer Ingelheim, Grifols, Novo Nordisk, Pfizer, and Sanofi Aventis, and has also acted as a consultant or ad hoc speaker/consultant for Bayer, Biotest, Boehringer Ingelheim, CSL Behring, Eli Lilly, Grifols, Novo Nordisk, Pfizer, and Sanofi Aventis. R. d'Oiron received fees or honoraria for attending advisory boards or speaking at symposia for Baxter, Bayer, CSL Behring, Novo Nordisk, Pfizer, and SOBI.

\section{Acknowledgments}

Editorial assistance was provided by Lisa Weissbach (LBT) and Mark Simmonds (Parexel) and the study was financially supported by Novo Nordisk Health Care AG. The authors also thank Soraya Benchikh el Fegoun (Novo Nordisk Health Care AG) for comments and advice.

Novo Nordisk Health Care AG financially supported the Glanzmann Thrombasthenia Registry in compliance with international guidelines for Good Publication Practice. Novo Nordisk Health Care AG also supported several authors' meetings held to plan and discuss the article and its content.

\section{References}

1 Bellucci S, Caen J. Molecular basis of Glanzmann's thrombasthenia and current strategies in treatment. Blood Rev 2002;16(03): 193-202
2 George JN, Caen JP, Nurden AT. Glanzmann's thrombasthenia: the spectrum of clinical disease. Blood 1990;75(07):1383-1395

3 Toogeh G, Sharifian R, Lak M, Safaee R, Artoni A, Peyvandi F. Presentation and pattern of symptoms in 382 patients with Glanzmann thrombasthenia in Iran. Am J Hematol 2004;77(02): 198-199

4 Poon MC, D'Oiron R, Von Depka M, et al; International Data Collection on Recombinant Factor VIla and Congenital Platelet Disorders Study Group. Prophylactic and therapeutic recombinant factor VIIa administration to patients with Glanzmann's thrombasthenia: results of an international survey. JThromb Haemost 2004;2(07):1096-1103

5 Di Minno G, Zotz RB, d'Oiron R, Bindslev N, Di Minno MN, Poon MC; Glanzmann Thrombasthenia Registry Investigators. The international, prospective Glanzmann Thrombasthenia Registry: treatment modalities and outcomes of non-surgical bleeding episodes in patients with Glanzmann thrombasthenia. Haematologica 2015;100(08):1031-1037

6 Poon MC, d'Oiron R, Zotz RB, Bindslev N, Di Minno MN, Di Minno G; Glanzmann Thrombasthenia Registry Investigators. The international, prospective Glanzmann Thrombasthenia Registry: treatment and outcomes in surgical intervention. Haematologica 2015;100(08):1038-1044

7 Novo Nordisk. NovoSeven summary of product characteristics. Available at: http://www.ema.europa.eu/docs/en_GB/document_library/EPAR_-_Product_Information/human/000074/ WC500030873.pdf. Accessed April 4, 2016

8 Brand A, Claas FH, Voogt PJ, Wasser MN, Eernisse JG. Alloimmunization after leukocyte-depleted multiple random donor platelet transfusions. Vox Sang 1988;54(03):160-166

9 Legler TJ, Fischer I, Dittmann J, et al. Frequency and causes of refractoriness in multiply transfused patients. Ann Hematol 1997;74(04):185-189

10 Trial to Reduce Alloimmunization to Platelets Study Group. Leukocyte reduction and ultraviolet B irradiation of platelets to prevent alloimmunization and refractoriness to platelet transfusions. N Engl J Med 1997;337(26):1861-1869

11 Ito K, Yoshida H, Hatoyama H, et al. Antibody removal therapy used successfully at delivery of a pregnant patient with Glanzmann's thrombasthenia and multiple anti-platelet antibodies. Vox Sang 1991;61(01):40-46

12 Vivier M, Treisser A, Naett M, et al. Glanzmann's thrombasthenia and pregnancy. Contribution of plasma exchange before scheduled cesarean section [in French]. JGynecol Obstet Biol Reprod (Paris) 1989;18(04):507-513

13 Martin I, Kriaa F, Proulle V, et al. Protein A Sepharose immunoadsorption can restore the efficacy of platelet concentrates in patients with Glanzmann's thrombasthenia and anti-glycoprotein IIb-IIIa antibodies. Br J Haematol 2002;119(04):991-997

14 NiaStase $\mathrm{RT}^{\circledR}$. Product Monograph. Available at: http://www. novonordisk.ca/content/dam/Canada/AFFILIATE/www-novonordisk-ca/OurProducts/PDF/niastase-product-monograph.pdf. Accessed November 8, 2017

15 Schulman S, Kearon C; Subcommittee on Control of Anticoagulation of the Scientific and Standardization Committee of the International Society on Thrombosis and Haemostasis. Definition of major bleeding in clinical investigations of antihemostatic medicinal products in non-surgical patients. JThromb Haemost 2005;3(04):692-694

16 Chitlur M, Ewing N, Kraut EH, et al. Recombinant factor VIla (rFVIIa) use in Glanzmann's thrombasthenia (GT) and other platelet disorders (OPDS): Hemophilia and Thrombosis Research Society (HTRS) registry data. [abstract]. J Thromb Haemost 2011; 9(Suppl. 2):340

17 Lak M, Scharling B, Blemings A, et al. Evaluation of rFVIIa (NovoSeven) in Glanzmann patients with thromboelastogram. Haemophilia 2008;14(01):103-110 